\title{
Biomarkers of tobacco smoke: the focus of a new 4-year study
}

The Center of Excellence in Environmental Toxicology (CEET), at the University of Pennsylvania School of Medicine, is to embark upon a 4-year study aiming to identify biomarkers of cigarette smoke exposure. The US\$2.3 million funding for the research has been awarded by the NIH as part of the Genes, Environment and Health Initiative. The study compliments current research being conducted by the CEET concerning gene-environment interactions in lung cancer, a venture which began earlier this year following a US $\$ 4$ million grant provided by the Pennsylvania Department of Health.

\section{'The study compliments current research being conducted by the CEET concerning gene-environment interactions in lung cancer...'}

The WHO state that one in ten adults worldwide are killed as a result of tobacco usage and it is listed as a leading cause of death in the USA. Earlier studies into the effects of smoking have found alterations within both the tissue and DNA of smokers, as well as those who are exposed to smoke. This study aims to research these findings on a large scale, with the use of reliable biomarkers. The study's investigators will conduct screening for biomarkers in smokers, nonsmokers as well as those regularly exposed to smoke in a second-hand capacity.

The investigators hope to develop a series of biomarkers that will test for smoke exposure, allowing nonsmokers and disease-free smokers to be distinguished from one another. It is thought that this data will enable subsequent studies into tobacco-related diseases to accurately assess cigarette smoke exposure in terms of incidence and genetic susceptibility, by using this study as a reference point.

"Only one in ten smokers get lung cancer, but the 5-year survival rate after diagnosis is only $15 \%$," stated the director of the CEET, Trevor M Penning. "The question is, how can we intervene earlier to identify people most at risk. We aim to look at the interaction of genetic susceptibility to lung cancer and biomarkers of exposure to cigarette smoke. At the end of the day, if we study genetics and exposure together, we'll hopefully have a very strong statement to say who is most at risk".

'The investigators hope to develop a series of biomarkers that will test for smoke exposure...'

Associate Professor of biostatistics at the University of Pennsylvania, Andrea Troxel, will be responsible for determining the applicability of biomarkers. Director of the Penn Microarray Core Facility, Don Baldwin, will also collaborate on this project, utilizing a discovery program to enable the identification of potential protein iomarkers in addition to conventional DNA and lipid biomarkers.

Commenting on the potential repercussions of the study, lead study investigator, Ian Blair enthused, "This could be like measuring cholesterol for preventing heart disease. If, for example, a certain chemical change is seen in a smoker's DNA, it might inspire them to give up smoking. You could show them a biomarker panel and say, 'Here's your DNA on smoking and here's DNA without smoking'. This is my vision".
Another institute to benefit financially from the NIH's Gene and Environment Initiative is the Pacific Northwest National Laboratory (PNNL), where a Center for Novel Biomarkers of Response is being established. Research at the PNNL will also focus on cigarette smoke, aiming to identify biomarkers for the systemic stress caused as a result of tobacco smoke exposure, studying obesity as a confounding factor. The center will yield a database of response biomarkers and researchers working on the project aim to develop a novel way to enable biomarkers to be measured at the point of care by utilizing nanotechnology-based sensors.

Sources: University of Pennsylvania Press Release. www.uphs.epenn.edu;

Pacific Northwest National Laboratory Press Release. www.pnl.gov 


\section{in brief..}

New biomarkers may improve diagnostic approaches for acute kidney injury. Waikar SS, Bonventre JV: Curr. Opin. Nephrol. Hypertens. 16(6), 557-564 (2007).

Urine may contain markers that are both sensitive and specific indicators of kidney injury, a new study reveals. $\mathrm{N}$-acetyl- $\beta$-D-glucosaminidase, neutrophil gelatinase-associated lipocalin, kidney injury molecule-1 and IL-18 are all listed as potential targets. Current diagnostic tests of acute kidney injury utilize blood urea nitrogen and serum creatine tests, which have been in use for many years. The discovery of new biomarkers for acute kidney injury will improve the capacity for future diagnostic approaches. Clinical studies of multiple biomarkers are required before these biomarkers can be adopted in a clinical setting

Biomarker for bladder cancer identified. M arguilis V, Lotan Y, Shariat SF: World J. Urol. (2007) (Epub ahead of print).

Research has indicated that Survivin may be a key biomarker in the diagnosis of bladder cancer. Preclinical bladder tumor models have demonstrated that inhibition of Survivin expression and/or function results in obstruction of tumor proliferation, as well as causing an increase in chemotherapy induced apoptosis. Large prosective trials are needed to confirm these results. Furthermore, for Survivin to be effectively exploited, development of appropriate detection assays will be required.

IFN- $\gamma$ may be a potential biomarker for virus exposure.

Li L, Dafour AP: Curr. Microbiol. (2007) (Epub ahead of print).

$B A L B / C$ mice were injected intraperitoneally with coxsackievirus B3 (CVB3) or coxsackievirus B4 (CVB4) diluted in sterilized phosphate-buffered saline (PBS). Mice in the control group were injected with PBS only. After 4 months, spleen cells from the mice were assayed for IFN- $\gamma$ release by memory T cells, following in vitro stimulation with viral antigens, phytohemagglutinin and PBS, respectively. Results of the study showed that memory T-cells from CVB3- infected mice incubated with CVB3 virus, caused a significant increase in IFN- $\gamma$; however, this did not occur after incubation with CV4 or PBS. M emory T cells from mice injected with CVB4 produced significant levels of IFN- $\gamma$ after incubation with CVB4, but not after incubation with CVB3 and PBS. The study provides evidence for a virus-specific nature of IFN$\gamma$ produced by memory $T$ cells and should be followed up by human studies in order to explore the potential for IFN- $\gamma$ as a biomarker of viral pathogen exposure.

\section{White blood cell count may be linked to cancer risk}

A recent study investigating links between the immune system and cancer has suggested that high levels of white blood cells (WBC) may indicate an increased risk of cancer in postmenopausal women.

143,748 postmenopausal women, free of cancer and aged between 50 and 79 years were enrolled in the study, which lasted over 7.8 years. Previousstudies have suggested an association between cancer and inflammation of the immune system; however, findings have remained inconclusive.

The results of the study revealed that women in the highest quartile for WBC (6.80-15.00 × $10^{9}$ cells/l) were $15 \%$ more likely to develop breast cancer, $19 \%$ more likely to develop colorectal cancer, $42 \%$ more likely to develop endome trial cancer and $62 \%$ more likely to develop lung cancer than those in the lowest quartile for WBC (2.50-4.79 × $10^{9}$ cells/I).

"Before these findings can be applied clinically, they should be replicated in other populations, with two measurements of $\mathrm{WBC}$ count, and expanded to include other biomarkers of inflammation that may be more specifically linked to underlying causal mechanisms of neoplasia," study researchers conclude.

Source: $M$ argolis $K L$, Rodabough RJ, Thomson $C A \&$ al.: Prospective study of leucocyte count as a predictor of incident breast, colorectal, endometrial, and lung cancer and mortality in postmenopausal women. Arch. Intern. Med. 167(17), 1837-44 (2007)

\section{Biomarker for coronary heart disease is reduced by a calorie-controlled diet}

A recent study has revealed that levels of lipoprotein-associated cholesterol (Lp-PL)A2, a marker associated with coronary heart disease, may be positively affected by a low-calorie diet.

Lp-PLA $A_{2}$ is a $\mathrm{Ca}^{2+}$-independent phospholipase commonly associated in plasma with low density lipoproteins (LDL) and particularly small, dense LDL (sdLDL) particles.

Alexandros Tselepis of the University of Ioannina, Greece, and coauthors, studied 28, nondiabetic obese women, recording glucose metabolism, lipid profile and Lp$\mathrm{PLA}_{2}$ activity at the onset of the study, and again after 4 months of maintaining a calorie-controlled diet. A $10 \%$ weight reduction reduced levels of $L p-P L A_{2}$, as well as the cholesterol content of veryLDL-cholesterol (LDL-C) and large, buoyant LDL-C particles. H owever, the study also showed that the cholesterol levels of sdLDL particles and the percentage proportion of sdLD L-C particles over the total LDL-C did not change, leading researchers to conclude that that they are affected by a mechanism different to that which alters levels of $L p-P L A_{2}$.

Study investigators comment, "T he present study shows for the first time that a low-calorie diet associated with weight loss in obese women reduces the plasma levels of Lp-PLA2, the novel inflammatory biomarker of coronary heart disease".

Source: Tzotzas T, Filippatos T D, Triantos $A \notin$ al.: Lp-PLA2 levels decrease significantly following calorie-controlled diet. Nutr. Metab. Cardiovasc Dis (2007) ([Epub ahead of print) 


\section{Biomarker panel may provide early detection of endometrial cancer}

A recent study investigating biomarkers for endometrial cancer has discovered a method that may enable early detection of endometrial cancer. Researchers from the University of Pittsburgh, USA, examined women with stages I-III endometrial cancer age-matched to healthy women. A panel of 64 biomarkers in patients' sera were analyzed, using the multiplex XM AP ${ }^{\mathrm{TM}}$ bead-based immunoassay.

The results of the study demonstrated patients with endometrial cancer to have significantly different patterns of expression of several serum biomarkers; CA125, CA15-3 and CEA were shown to be higher in patients with stage III endometrial cancer, in comparison with those with stage I. With $98.3 \%$ sensitivity and $98.0 \%$ specificity, prolactin was the strongest discriminating biomarker for endometrial cancer. However, when used on its own, prolactin was not cancer selective. By combining prolactin with the biomarkers $\mathrm{GH}$, Eotaxin, E-selectin and TSH, both sensitivity and specificity were greatly enhanced, allowing endometrial, ovarian and breast cancers to be distinguished from one another.

Logistic regression, separating hyperplane, $\mathrm{k} N$ earest $\mathrm{N}$ eighbours and classification tree were all used by investigators as methods of classification. Cross validation was conducted in which sera from 115 patients with endometrial cancer was compared with that of 135 healthy patients for each classification method. When the five biomarkers were combined, they were able to distinguish between endometrial and ovarian cancer with a sensitivity and specificity of $83-97 \%$ and $89-95 \%$ respectively; endometrial and breast cancer were distinguised with a sensitivity and specificity of $89-90 \%$ and $94-97 \%$, respectively. Results varied according to the classification system.

The researchers believe that the multimarker panel may provide early detection of endometrial cancer, preventing late-stage discovery that often results in high levels of morbidity and mortality. The researchers conclude, "T his multimarker panel may present a prototype of a clinical screening test for women with endometrial cancer who do not have common endometrial cancerassociated symptoms".

Source: Yurkovetsky Z, Ta'asan S, Steve Skates S \& al.: D evelopment of multimarker panel for early detection of endometrial cancer. $\mathrm{H}$ igh diagnostic power of prolactin. Gyneed. Oncd. 107(1), 58-65 (2007).

\section{Cyclooxygenase-2: potential biomarker for cancer prevention}

Cylooxgenase-2 (COX-2) represents a potential target for cancer prevention, researchers say, following a study into its role in cervical cancer. The study is one of many that show the enzyme to be upregulated in a number of cancers. Researchers examined 47 abnormal and normal cervical biopsies from patients between 18-65 years of age; abnormal and normal biopsies were paired and assessed for high-risk human papillomavirus (HPV) as well as inflammation, histopathological diagnosis and COX-2 protein concentration by ELISA.

"If cervical inflammation promotes the progression of H PV infection from a premalignant phase to cervical cancer, then ELISA may provide a feasible, cost-effective alternative to assess COX-2 expression ... before the development of persistent HPV infection and cervical cancer," commented study investigator Salvador Saldivar and coauthors.
Results of the study showed COX-2 proteins to be 4.9-times greater in abnormal than normal biopsies. Furthermore, a comparison of cervical areas from the same patient showed levels of COX-2 to be significantly higher (average $403.7 \mathrm{ng} \mathrm{COX}-2 / \mathrm{mg}$ protein) compared with normal areas (average $83.1 \mathrm{ng} \mathrm{COX}-2 / \mathrm{mg}$ protein).

Although no significant association was shown between COX-2 and H PV high-risk positivity, it may be that the small sample size was a limiting factor.
The researchers conclude that their study is suggestive of COX-2 induction in a premalignant phase of cervical carcinogenesis, correlated with inflammation. Additional, larger trials are needed to confirm the results of this study and to improve current understanding of the biomarker potential of COX-2.

Source: Saldivar JS, Lopez D, Feldman R \& al.: COX-2 overexpression as a biomarker for early cervical carcinogenesis: a pilot study. Gyneed. Oncd. 107(1), S155-S162 (2007).

\section{About the Bulletin Board}

The Bulletin Board highlights some of the most important events and launches in the application of biomarkers in medicine. The editorial team welcomes suggestions for timely, relevant items. If you have newsworthy information, please contact:

Laura Dormer, Head of Commissioning

Future Medicine Ltd, Unitec House,

2 Albert Place, London N3 1QB, UK

Tel.: +44 (0)20 8371 6090; Fax: +44 (0)20 83432313

E-mail: I.dormer@futuremedicine.com 